\author{
Military Technical College \\ Kobry El-Kobbah, \\ Cairo, Egypt.
}

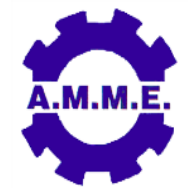

$18^{\text {th }}$ International Conference on Applied Mechanics and Mechanical Engineering.

\title{
UNCERTAINTY ESTIMATION FOR ROTOR VIBRATION AMPLITUDE MEASURING SYSTEM
}

\author{
M. Makram*, S. S. Kossa*, M. K. Khalil", A. F. Nemnem and S. Wagdy
}

\begin{abstract}
This study represents a measurement uncertainty model for rotor vibration amplitude measuring system. The measuring system is established to provide a simple way for rotor vibration measurement and consists of a combination of different sensors. A simple data acquisition software is resolved using LabVIEW. This program enables displaying and recording the directly measured data. Type-A and type-B uncertainties are estimated in detail for measuring results. The two different types are combined to evaluate the deviation of readings resulted from several sources. The overall system accuracy for rotor vibration amplitude is derived from combining uncertainties of the two measured perpendicular vibration displacements. An error bar is provided for each reading to indicate the possible deviation with $95 \%$ level of confidence. Uncertainty detailed calculation for rotor vibration amplitude measuring system is computed as a function of the directly measured values.
\end{abstract}

\section{KEY WORDS}

Accompanied uncertainty, System overall accuracy, Measuring error, Standard uncertainty. 


\section{INTRODUCTION}

An accurate measurement is one that is close to the true value which is unknown. Measurement Uncertainty is used to show the expected range that of the true value. While this term was provided for many of the high accuracy measurements, it is required that measurement uncertainty be known and consistent with required measurement efficiency of any screening or measuring device. Uncertainty is the value provided with a measured value that indicates, according to a specified level of confidence, the range expected to include the true measurement result [1].

It is not acceptable, in several fields, to present experimental results without calculating its uncertainties. A common description of error sources and uncertainty in experimental measurements is presented by Moffat [2], with a numerical technique for calculating uncertainty for computerized data.

Uncertainty analysis reports are an important for measurement quality assurance. The analysis report is recommended to be comprehensive enough to describe a functional guide for measurements reading [3]. In 2010, Suzanne Castrup [4] recommended workouts for calculating measurement uncertainty. An example analysis report is provided to show the collection of error elements to determine uncertainty ranges. In addition, the data and information used were introduced.

\section{MEASURING SYSTEM DESCRIBTION}

The measuring system is used to investigate the automatic ball balancer (ABB) effect on a small rotor vibration [5]. ABB is a passive balancing device that consists of several metal balls rotating freely in a circular track. ABB is a good solution for reducing rotor vibration produced from imbalance, specially at high speeds. Rotor vibration is required to be measured in sensitivity of $0.01 \mathrm{~m}$ [6].

In the used model shown in Fig. 1, the rotor consists of an elastic $6 \mathrm{~mm}$ diameter shaft made of high strength steel. One steel disc is clamped at the shaft midspan. The speed measurement takes place via two orthogonal inductive sensors clamped as shown in Fig. 2. Rotor kit is vertically supported on a test rig using four screw bolts with rubber spacers for vibration damping not to transfer the resulted vibration to the rig

A four balls $A B B$ is designed and manufactured according to the rotor parameters. It is linked to the rotor for avoiding any relative rotation. ABB design enables the usage of balls of different diameters and masses. Two bolts clamp the imbalance mass as shown in Fig. 3.

A data acquisition system is built up to enable a simple way in vibration measurement. The system consists of: two inductive non-contact displacement sensors connected to the sensor supply module TM 151 then to two NI 9223 BNC slots fixed in the CDAQ-1988 then to PC via USB port.

A simple program is resolved using LabVIEW software, this program enables displaying and recording the received data in excel sheet to be easily processed. 


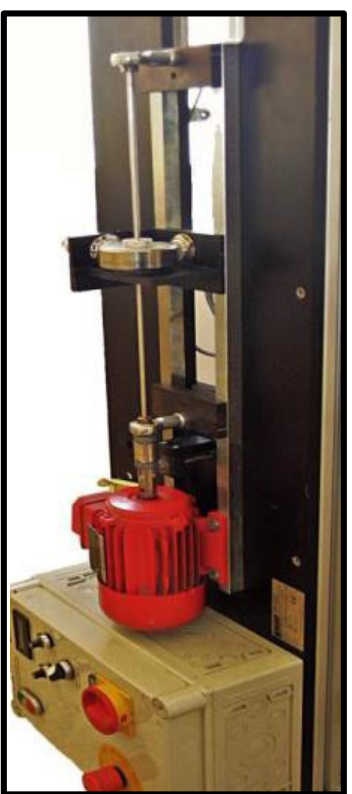

Fig. 1. Manufacture ABB.

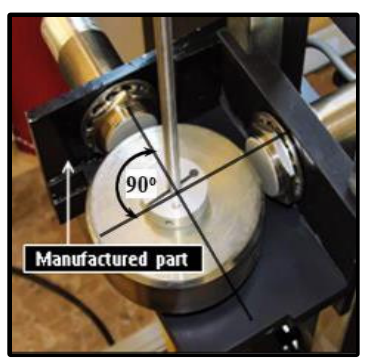

Fig. 2. Sensors clamping.

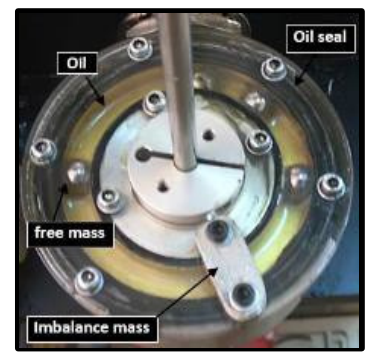

Fig. 3. Manufactured ABB.

Data is processed as shown in Fig. 4. After getting displacement sensor calibration curve, sensor transfer function is deduced. In addition, the rotor response curve, which shows a relation between angular speed and vibration amplitudes, is drawn by getting the vibration maximum amplitude at several speeds.

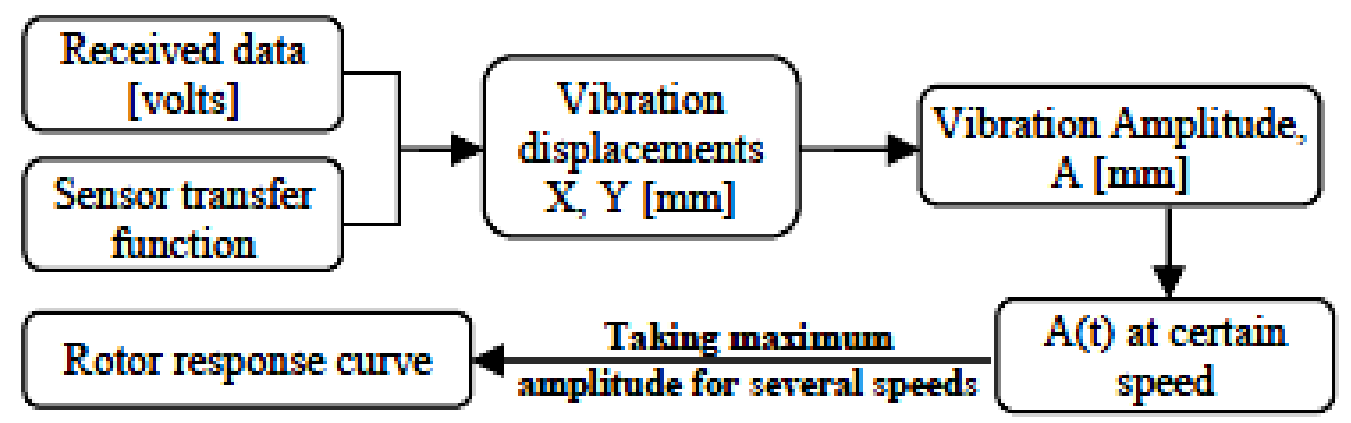

Fig. 4. Data Processing Flow Chart.

\section{MEASUREMENT UNCERTAINTY}

Uncertainty can be divided to two different types [1]; Type A and Type-B. Type-A uncertainty analysis is calculated statistically, from frequent measurements. Type-B uncertainty analysis is worked out using sensors' specifications and not calculated statistically as Type-A. Both uncertainties will be combined to provide a number called a standard uncertainty.

$$
A=\sqrt{X^{2}+Y^{2}}
$$

For example; the measurement of amplitude at $404 \mathrm{rad} / \mathrm{sec}$ of the rotor is presented in this study. The amplitude vibration is the square root of the summation of squares of displacements in $\mathrm{x}$ and $\mathrm{y}$ directions. So, that two displacement sensors were fixed at two orthogonal directions to measure the vibration displacements in each direction, 
three confirmed readings are taken in each measurement. Specified measurement procedures are followed to ensure that all measurements are the clearance between the disc and the safety bearing are ensured when operating the rotor every time.

\section{Type-A Uncertainty $\left(U_{A}\right)$ Calculation}

If an uncertainty is based on a statistical analysis, it is to be treated as a Type-A uncertainty. Most random uncertainties are Type A uncertainties. They vary each time a measurement is made. The uncertainty can be reduced by averaging lots of measurements, but it can never be totally eliminated. In this study, only one of several measurements is presented, three readings are taken for each measurement due to the great number of measurements. However, more readings are preferred. The more nearly equal readings you take, the less Type-A uncertainty you obtain. For Type-A uncertainty analysis, the changeability of $n$-readings is characterized by their standard deviation $(\sigma)$, as shown in Fig. 5, given by Eqn. (2).

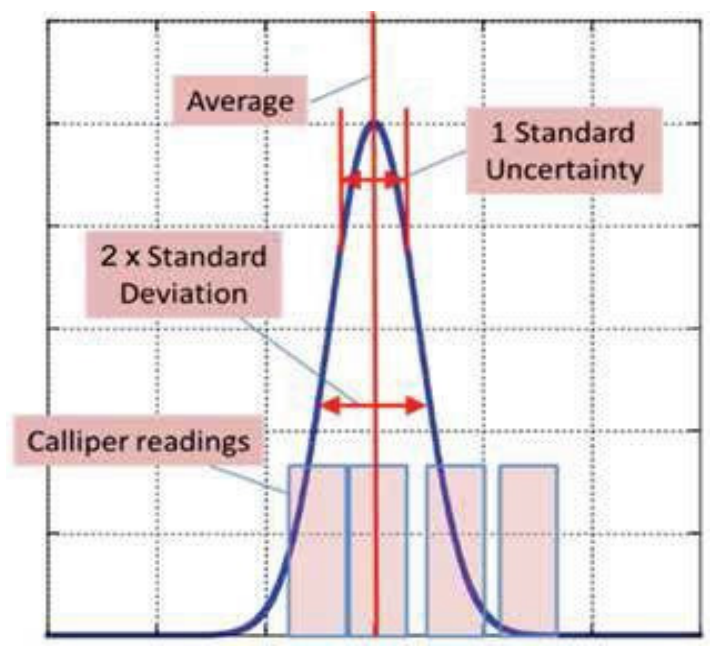

Fig. 5. Type-A uncertainty [1].

$$
\sigma=\sqrt{\frac{\sum_{i=1}^{n}\left(\text { reading }_{i}-\text { average }^{2}\right.}{n-1}}
$$

On measuring X-displacement, the three confirmed readings taken were -0.3936 , -0.39798 , and -0.39809 , with mean value of $-0.3966 \mathrm{~mm}$, so standard deviation in $x$-direction will be $0.002582 \mathrm{~mm}$. While on measuring $y$-displacement, the three confirmed readings taken were $-0.1532,-0.1424$, and $-0.1482 \mathrm{~mm}$, with mean value of $-0.1479 \mathrm{~mm}$, so standard deviation in $\mathrm{x}$-direction will be $0.00539 \mathrm{~mm}$.

Table 1 and Table 2 show the steps for calculating the standard deviation for $\mathrm{x}$ and $\mathrm{y}$ vibration displacements. The standard deviation of $0.00258 \mathrm{~mm}$ is an estimate for the above X-displacement readings. While it is $0.00539 \mathrm{~mm}$ for the Y-displacement readings. Taking more readings would improve the confidence in the estimate of the average. For $n$ readings, this fact is defined by terms of the standard uncertainty (UA) provided with the mean as shown in Eqn. (3). 
Table 1. Type-A uncertainty calculation for X-displacement.

\begin{tabular}{|c|c|c|c|}
\hline$\underline{\text { Measurement }}$ & $\underline{\text { Reading }[\mathrm{mm}]}$ & (Reading-Average) & (Reading-Average) 2 \\
\hline $1-$ & -0.39356375 & 0.002979896 & 0.000009 \\
\hline $2-$ & -0.397982187 & -0.001438542 & 0.000002 \\
\hline $3-$ & -0.398085 & -0.001541354 & 0.000002 \\
\hline average $=$ & -0.396543646 & sum $=$ & 0.000013 \\
\hline & & $\operatorname{sum} / 2=$ & 0.000007 \\
\hline & ndard deviation $=$ & $V(\operatorname{sum} / 2)=$ & 0.002581177 \\
\hline Type A & indard uncertainty $=$ & standard deviation $/ \mathrm{V} n=$ & 0.001490243 \\
\hline
\end{tabular}

Table 2. Type-A uncertainty calculation for $Y$-displacement.

\begin{tabular}{|c|c|c|c|}
\hline$\underline{\text { Measurement }}$ & $\underline{\text { Reading }[\mathrm{mm}]}$ & (Reading-Average) & (Reading-Average) 2 \\
\hline $1-$ & -0.15319875 & -0.00527625 & 0.000028 \\
\hline $2-$ & -0.142408438 & 0.005514062 & 0.000030 \\
\hline $3-$ & -0.148160313 & -0.000237812 & 0.000000 \\
\hline \multirow[t]{2}{*}{ average $=$} & -0.1479225 & sum $=$ & 0.000058 \\
\hline & & $\operatorname{sum} / 2=$ & 0.000029 \\
\hline \multicolumn{2}{|c|}{ standard deviation $=$} & $V(\operatorname{sum} / 2)=$ & 0.005399086 \\
\hline \multicolumn{2}{|c|}{ Type A standard uncertainty $=$} & standard deviation $/ \mathrm{V} \mathrm{n}=$ & 0.003117164 \\
\hline
\end{tabular}

$$
\begin{gathered}
\mathrm{U}_{A}=\frac{\sigma}{\sqrt{n}} \\
\mathrm{U}_{A_{X}}=0.00149 \mathrm{~mm} \quad, \quad \mathrm{U}_{A_{Y}}=0.00312 \mathrm{~mm}
\end{gathered}
$$

The standard uncertainty provided with the mean is calculated in both $\mathrm{X}$-direction and Y-direction. This uncertainty is grounded on the concept that the taken readings are resulted from a normal probability distribution. The three confirmed readings are worked out for calculating the standard uncertainty.

\section{Type-B Uncertainty $\left(U_{B}\right)$ Calculation}

Any uncertainty not based on a statistical analysis is to be treated as a Type-B uncertainty. Most systematic uncertainties are Type-B uncertainties. Type-B uncertainty calculation is required to assess uncertainties where there is measurement accuracy for the instrument or the sensor used, which affect the reading in the same behavior. Only the upper and lower limits are provided and described by a 'rectangular distribution' as shown in Fig. 6, in which the value is equally likely to fall anywhere within the interval. The two used sensors are identical. So, that Type-B uncertainty in measuring $X$ and $Y$ displacement are considered the same. To obtain Type-B uncertainty, it is required to use the rectangular distribution range to calculate a standard deviation then the standard uncertainty. To do this, the half range is divided by the square root of three (approximately 1.73) as presented in Eqn. (5). 


$$
\mathrm{U}_{B_{X, Y}}=\frac{\text { half the range }}{\sqrt{3}}=0.012 \mathrm{~mm}
$$

In the presented case, the calibration certificate states that the displacement sensor should read within $\pm 0.02 \mathrm{~mm}$ of the true value, if it is used correctly and the temperature is within the range $0^{\circ} \mathrm{C}$ to $40^{\circ} \mathrm{C}$. The standard uncertainty related to the device calibration is thus $0.012 \mathrm{~mm}$.

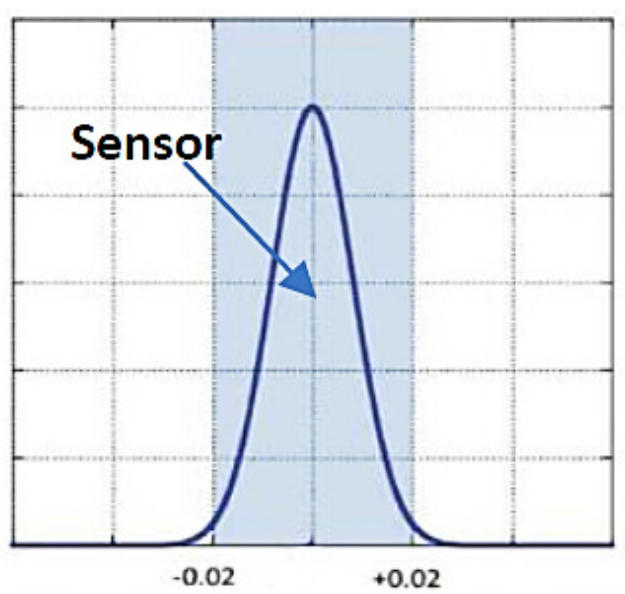

Fig. 6. Type-B uncertainty [1].

\section{COMBINED UNCERTAINTY CALCULATION}

After evaluating the individual standard uncertainties, the combined standard uncertainty is calculated by getting the root sum of its squares. The result is called the combined standard uncertainty.

$$
\begin{aligned}
& \mathrm{U}_{X}=\sqrt{\mathrm{U}_{A_{X}{ }^{2}+\mathrm{U}_{B_{X}}{ }^{2}}=0.0116 \mathrm{~mm}} \\
& \mathrm{U}_{Y}=\sqrt{{\mathrm{U}_{A_{Y}}{ }^{2}+\mathrm{U}_{B_{Y}}{ }^{2}}^{2}}=0.01196 \mathrm{~mm}
\end{aligned}
$$

Table 3. Combined uncertainty of X-displacement calculation.

\begin{tabular}{|c|c|c|c|c|}
\hline $\begin{array}{c}\text { Source of } \\
\text { Uncertainty }\end{array}$ & Value $[\mathrm{mm}]$ & $\begin{array}{c}\text { Probability } \\
\text { Distribution }\end{array}$ & Factor & $\begin{array}{c}\text { Standard } \\
\text { Uncertainty }[\mathrm{mm}]\end{array}$ \\
\hline variability & 0.0015 & Normal & 1 & 0.0015 \\
\hline calibration & \pm 0.02 & Rectangular & $1 / \mathrm{v} 3$ & 0.0115 \\
\hline \multicolumn{6}{|c|}{ Standard Uncertainty $=\sqrt{U_{A}^{2}+U_{B}^{2}}$} & 0.0116 \\
\hline
\end{tabular}

Table 4. Combined uncertainty of Y-displacement calculation.

\begin{tabular}{|c|c|c|c|c|}
\hline $\begin{array}{c}\text { Source of } \\
\text { Uncertainty }\end{array}$ & Value $[\mathrm{mm}]$ & $\begin{array}{c}\text { Probability } \\
\text { Distribution }\end{array}$ & Factor & $\begin{array}{c}\text { Standard } \\
\text { Uncertainty }[\mathrm{mm}]\end{array}$ \\
\hline variability & 0.0031 & Normal & 1 & 0.0031 \\
\hline calibration & \pm 0.02 & Rectangular & $1 / \sqrt{ } 3$ & 0.0115 \\
\hline \multicolumn{6}{|c|}{ Standard Uncertainty $=\sqrt{U_{A}^{2}+U_{B}^{2}}$} & 0.0120 \\
\hline
\end{tabular}


There are two terms that may cause a deviation of our reading. The first one is from the changeability of the data (Type-A), while the second one from the sensor calibration certificate (Type-B). So, in our case the two components are integrated as shown in Tables 3 and 4 . The best evaluation of the length is determined by getting the average of the 3 readings. The provided standard uncertainty is calculated by combining the standard uncertainties resembled the major factors which may lead the sensors to read incorrectly. Finally, the length and its provided standard uncertainty are estimated.

\section{OVERALL SYSTEM ACCURACY}

A measurement system is always built by several components, each component is subjected to individual inaccuracy. After calculating individual uncertainties, the overall system uncertainty $(U)$ is computed. In our experiments, the vibration amplitude value $(A)$ is computed from the two measured vibration displacements $X$ and $Y$ as shown in Eqn. (7).

$$
A=\sqrt{X^{2}+Y^{2}}
$$

For the overall system uncertainty analysis, consider the problem of computing a quantity $(A)$ which is a function of independent measured variables $X$ and $Y$. For small changes in the $X$ and $Y$ measured values at a given operating points, a good expression for the corresponding change in $\mathrm{A}$ is given using Taylor series expansion.

$$
\Delta A \approx \frac{\partial A}{\partial X} \cdot \Delta X+\frac{\partial A}{\partial Y} . \Delta Y
$$

The partial derivatives can be considered the sensitivity of amplitude value to be changed with respect to $X$ and $Y$ changes. For a small value of partial derivative, this means that $A$ is less sensitive to the change of corresponding value and vice versa. $\Delta \mathrm{X}$ and $\Delta \mathrm{Y}$ are considered uncertainties $\mathrm{UX}, \mathrm{UY}$ in each measured value $\mathrm{X}$ and $\mathrm{Y}$. So that, the corresponding overall uncertainty for rotor amplitude $\left(U_{\text {amp. }}\right)$ is computed, using Eqn. (9), as the root sum square $\left(r_{\mathrm{ss}}\right)$ formula.

$$
U_{\text {amp. }} \approx \sqrt{\sum_{i=1}^{n}\left(\frac{\partial A}{\partial x_{i}} \cdot U_{i}\right)^{2}}=\sqrt{\left(\frac{\partial A}{\partial X} \cdot U_{X}\right)^{2}+\left(\frac{\partial A}{\partial Y} \cdot U_{Y}\right)^{2}}
$$

Eqn. (9) is valid only if the input quantities $X$ and $Y$ are independent or uncorrelated. But in the presented case vibration displacements are recorded at certain operating point with fixed angular speed $(\omega)$. Then, $A$ is function of $(\omega)$ and this correlation is considered.

$$
A(t)=\frac{\omega^{2} e}{\sqrt{\left(\frac{k}{m}-\omega^{2}\right)^{2}+\left(\frac{c \omega}{m}\right)^{2}}}
$$

When the input quantities are correlated [7], the appropriate expression for the overall uncertainty $\left(\mathrm{U}_{\mathrm{amp}}\right.$.) provided with the measured result is derived as shown in Eqn. (11). 


$$
\begin{gathered}
U=\sqrt{\sum_{i=1}^{n} \sum_{j=1}^{n}\left(\frac{\partial A}{\partial x_{i}} \cdot \frac{\partial A}{\partial \omega} \cdot U_{i j}\right)^{2}}=\sqrt{\sum_{i=1}^{n}\left(\frac{\partial A}{\partial x_{i}} \cdot U_{i}\right)^{2}+2 \sum_{i=1}^{n-1}\left(\frac{\partial A}{\partial x_{i}} \cdot \frac{\partial A}{\partial x_{j}} \cdot U_{i j}\right)^{2}} \\
U_{\text {amp. }}=\sqrt{\left(\frac{\partial A}{\partial X} \cdot U_{X}\right)^{2}+\left(\frac{\partial A}{\partial Y} \cdot U_{Y}\right)^{2}+2\left(\frac{\partial A}{\partial X} \cdot \frac{\partial A}{\partial \omega} \cdot U_{X \omega}\right)^{2}+2\left(\frac{\partial A}{\partial Y} \cdot \frac{\partial A}{\partial \omega} \cdot U_{Y \omega}\right)^{2}}
\end{gathered}
$$

where $x_{i}$ estimates of $X$ and $Y$ displacements, $U_{i j}$ is the estimated covariance associated with $x_{i}$ and $x_{j}$. The degree of correlation between $x_{i}$ and $\omega$ is characterized by the estimated correlation coefficient $\left(r_{i j}\right)$.

$$
r_{i j}=\frac{U_{i j}}{U_{i} \cdot U_{j}}
$$

where $\mathrm{rij}=\mathrm{rji}$, and $-1 \leq \mathrm{rij} \leq+1$. If the estimates $\mathrm{xi}$ and $\mathrm{xj}$ are independent, $\mathrm{rij}=0$, and a change in one does not imply an expected change in the other. For the experiment, we consider ri $\omega=1$ for the considerable effect and the other partial derivatives are obtained.

$$
\begin{aligned}
\frac{\partial A}{\partial \omega}=\frac{2 \omega e \times \sqrt{\left(\frac{k}{m}-\omega^{2}\right)^{2}+\left(\frac{c \omega}{m}\right)^{2}}}{\left(\frac{k}{m}-\omega^{2}\right)^{2}+\left(\frac{c \omega}{m}\right)^{2}} & \frac{2(-2 \omega)\left(\frac{k}{m}-\omega^{2}\right)+2 c\left(\frac{c \omega}{m}\right)}{2 \times \sqrt{\left(\left(\frac{k}{m}-\omega^{2}\right)^{2}+\left(\frac{c \omega}{m}\right)^{2}\right)^{3}}}=0.000003 \\
\frac{\partial A}{\partial X} & =1 \\
\frac{\partial A}{\partial Y} & =1
\end{aligned}
$$

Then Eqn. (14) can be written as:

$$
\begin{gathered}
U_{\text {amp. }} \approx \sqrt{\left(\frac{\partial A}{\partial X} \cdot U_{X}\right)^{2}+\left(\frac{\partial A}{\partial Y} \cdot U_{Y}\right)^{2}+2\left(\frac{\partial A}{\partial X} \cdot \frac{\partial A}{\partial \omega} \cdot U_{X} \cdot U_{\omega}\right)^{2}+2\left(\frac{\partial A}{\partial Y} \cdot \frac{\partial A}{\partial \omega} \cdot U_{Y} \cdot U_{\omega}\right)^{2}} \\
=\sqrt{(0.0116)^{2}+(0.01196)^{2}+2(0.000003 \times 0.0116 \times 0.31416)^{2}+2(0.000003 \times 0.01196 \times 0.31416)^{2}}
\end{gathered}
$$$$
U_{\text {amp. }}=0.01669
$$

The effect of the connection between $X$ and $Y$ displacement with the angular speed is very small, as the small value of $\frac{\partial A}{\partial \omega}$ and the small error of the rpm sensor $\pm 3 \mathrm{rpm}$ which corresponds to $\pm 0.341 \mathrm{rad} / \mathrm{sec}$. This correlation is considered to provide a whole view of overall system uncertainty analysis. 


\section{EXPANDED UNCERTAINTY FOR HIGHER LEVEL OF CONFIDENCE}

The level of confidence can be increased if the standard uncertainty is multiplied by a coverage factor $(K)$ to give an expanded uncertainty $(U)$.

$$
U=K \times \text { overall uncertainty }
$$

Table 5. Expanded uncertainty for different levels of confidence.

\begin{tabular}{|c|c|c|c|}
\hline $\begin{array}{c}\text { Standard } \\
\text { Uncertainty }\end{array}$ & $\begin{array}{c}\text { Converage } \\
\text { Factor }\end{array}$ & $\begin{array}{c}\text { Expanded } \\
\text { Uncertainty }\end{array}$ & $\begin{array}{c}\text { Probability that true } \\
\text { value lies in range }\end{array}$ \\
\hline \multirow{3}{*}{0.016691441} & 1 & 0.016691441 & $68 \%$ \\
\cline { 2 - 4 } & 2 & 0.033382882 & $95 \%$ \\
\cline { 2 - 4 } & 3 & 0.050074323 & $99.8 \%$ \\
\hline
\end{tabular}

The confidence level is increased to $95 \%$ or even $99 \%$ by combining with the appropriate coverage factor as shown in Table 5. For the presented example of vibration amplitude measurement at $404 \mathrm{rad} / \mathrm{sec}$, it is written: $0.423235 \pm 0.033383$ $\mathrm{mm}$. The reported uncertainty is based on a standard uncertainty multiplied by coverage factor $\mathrm{K}=2$, providing a level of confidence of approximately $95 \%$.

\section{IMPORTANCE OF CALCULATING UNCERTAINTY RANGES}

From a previous study by Michael et al. [6], a comparison between the numerical and the experimental results is done as shown in Fig. 7. The numerical solution is represented by a continuous curve, where the points represent the experimental reading provided with error bars representing its uncertainty with $99.8 \%$ level of confidence. Zooming in the curve above the critical speed, it is observed that all the numerical results of the proposed model lie in the measured experimental ranges. For model validation curves, it is shown that the numerical results lie in the range of the calculated uncertainty provided with experimental readings. These curves ensure the importance of calculating measurement uncertainty.

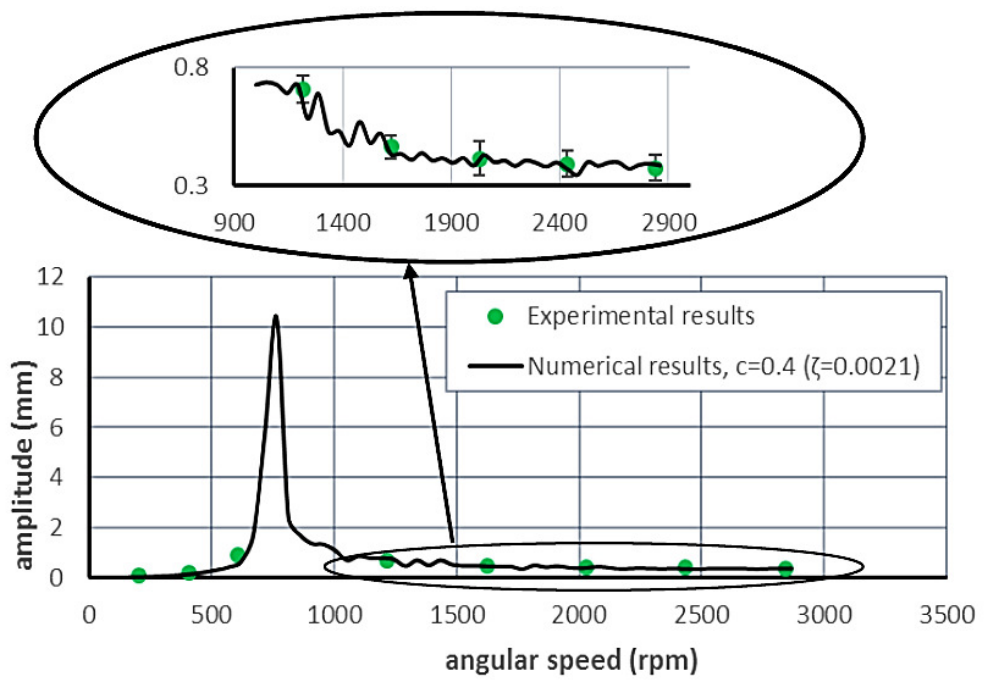

Fig. 7. Rotor without $A B B$ response curve. 


\section{CONCLUSION}

The uncertainty detailed calculation for any measuring system provides the true value range with a specified level of confidence and increase measurement reliability. Parametric studies including uncertainty ranges are more efficient to be considered. Uncertainty detailed calculation for rotor vibration amplitude measuring system is computed as a function of the measured values. Error bars are provided for each reading to show its uncertainty with $95 \%$ level of confidence.

\section{ACKNOWLEDGMENT}

The authors express their thanks to Dr./ Mohamed Y. Zakaria for his great contribution to this paper.

\section{REFERENCES}

[1] Evaluation of measurement data - Guide to the expression of uncertainty in measurement," JCGM, 2008.

[2] R. J. Moffat, "Describing the uncertainties in experimental results," Experimental Thermal and Fluid Science, vol. 1, pp. 3-17, 1988/01/01/ 1988.

[3] t. U. S. G. t. t. E. o. U. i. ANSI/NCSL Z540-2-1997 and Measurement.

[4] S. Castrup, "Important Elements of an Uncertainty Analysis Report," NCSL International Workshop and Symposium, 2010.

[5] M. Makram et al., "Experimental Investigation of Automatic Ball Balancer Effect on Unbalanced Rotor Vibration," The IV International AMMCS Interdisciplinary Conference Waterloo, Ontario, Canada, 2017.

[6] M. Makram, et al., "Effect of Automatic ball balancer on unbalanced rotor vibration," ASAT - 17 - Military Technical College, Cairo, EGYPT, 2017.

[7] Evaluation of measurement data- Guide to the expression of uncertainty in measurement (2008).

[8] E. O. Doebelin, Measurement systems: applications and design, Mac Graw-Hill, 2004. 